\title{
Efficient Multi-keV X-Ray Sources from Ti-Doped Aerogel Targets
}

K.B. Fournier, C. Constantin, G. Gregori, M.C. Miller, C.A. Back, L.J. Suter, J. Davis and J. Grun

This article was submitted to the proceedings of the $14^{\text {th }}$ APS Topical Conference on Atomic Processes in Plasmas

16 April, 2004

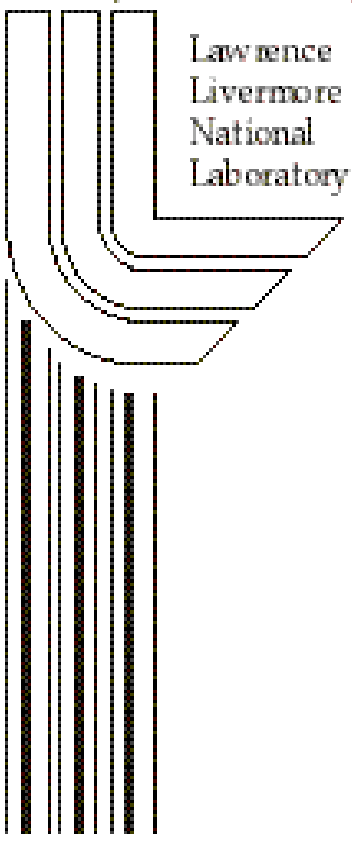




\section{DISCLAIMER}

This document was prepared as an account of work sponsored by an agency of the United States Government. Neither the United States Government nor the University of California nor any of their employees, makes any warranty, express or implied, or assumes any legal liability or responsibility for the accuracy, completeness, or usefulness of any information, apparatus, product, or process disclosed, or represents that its use would not infringe privately owned rights. Reference herein to any specific commercial product, process, or service by trade name, trademark, manufacturer, or otherwise, does not necessarily constitute or imply its endorsement, recommendation, or favoring by the United States Government or the University of California. The views and opinions of authors expressed herein do not necessarily state or reflect those of the United States Government or the University of California, and shall not be used for advertising or product endorsement purposes.

This is a preprint of a paper intended for publication in a journal or proceedings. Since changes may be made before publication, this preprint is made available with the understanding that it will not be cited or reproduced without the permission of the author.

\section{Auspices}

This work was performed under the auspices of the U.S. Department of Energy by University of California Lawrence Livermore National Laboratory under contract No. W-7405-Eng-48. 


\title{
Efficient multi-keV x-ray sources from Ti-doped aerogel targets
}

\author{
K. B. Fournier*, C. Constantin*, G. Gregori*, M. C. Miller*†, C. A. Back*, \\ L. J. Suter*, J. Davis** and J. Grun ${ }^{\ddagger}$ \\ ${ }^{*}$ Lawrence Livermore National Laboratory, P.O. Box 808, L-41, Livermore, CA 94550, USA \\ ${ }^{\dagger}$ Los Alamos National Laboratory, P.O. Box 1663 MS E540, Los Alamos, NM 87545 \\ ${ }^{* *}$ Alme \& Associates, 6020 Richmond Highway, Alexandria, VA 22303 USA \\ ¥Plasma Physics Division, Naval Research Laboratory, 4555 Overlook Ave., SW, Washington DC \\ 20375 USA
}

\begin{abstract}
We have measured the production of $h v \approx 4.7 \mathrm{keV}$ x-rays from low-density Ti-doped aerogel ( $\rho \approx 3 \mathrm{mg} / \mathrm{cc}$ ) targets at the OMEGA laser facility (University of Rochester), with the goal of maximizing $\mathrm{x}$-ray output. Forty OMEGA beams $\left(\lambda_{\mathrm{I}}=0.351 \mu \mathrm{m}\right)$ illuminated the two cylindrical faces of the target with a total power that ranged from 7 to $14 \mathrm{TW}$. The laser fully ionizes the target $\left(n_{e} / n_{\text {crit }} \leq 0.1\right)$, and a laser-bleaching wave excites, supersonically, the high-Z emitter ions in the sample. Heating in the target was imaged with gated $\mathrm{x}$-ray framing cameras and an X-ray streak camera. Ti K-shell $\mathrm{x}$-ray emission was spectrally resolved with a two-channel crystal spectrometer and also with a set of filtered aluminum $\mathrm{x}$-ray diodes, both instruments provide absolute measurement of the multi-keV x-ray emission. We find between $40-260 \mathrm{~J}$ of output with $4.67 \leq h v \leq 5.0 \mathrm{keV}$. Radiation-hydrodynamic calculations predict late time enhancement of the $\mathrm{x}$-ray power due first to axial stagnation of the heating waves, then, ablatively-driven radial compression from the target walls.
\end{abstract}

\section{INTRODUCTION}

Maximizing the conversion efficiency (CE) of laser energy into multi-keV $\mathrm{x}$-rays is a general concern to many areas of high-energy-density plasma physics [1-4]. Bright $\mathrm{x}$-ray sources are needed for backlighters in order to radiograph targets in inertialconfinement fusion experiments (ICF) $[3,4]$. As the targets get larger, and as compression in the targets increases, the backlighter sources need to be brighter and the backlighter photon energies must increase. To this end, for a given laser power, backlighters can become brighter by becoming more efficient at converting the drive beams to multi-keV x-rays. Volumetric heating of low-density gas targets has been shown to be a very efficient method of producing $x$-rays [5]. However, gas targets are limited to a few photon energies (Ar K-shell: $3.1 \mathrm{keV}, \mathrm{Kr}$ L-shell: $1.8 \mathrm{keV}$, Xe L-shell: $4.5 \mathrm{keV}$, and $\mathrm{Kr}$ K-shell: $13.3 \mathrm{keV}$ ). For a given laser power, the CE also falls off sharply with increasing output X-ray energy [6]. X-ray-yield scaling in solid targets has been studied as a function of laser energy, laser wavelength, laser-pulse length and focusing properties [7-13]. Historically, solid targets, usually massive disks, have demonstrated efficiencies of only fractions of a percent for multi-keV photon energies. The efficiencies of these massive targets are compromised because much of the laser energy is deposited in a (relatively) 
low-density region at the critical surface, while the region of the target dominating the radiation output may be a higher-density region that must be conductively heated; steep gradients in solid targets make optimization of the multi-keV source difficult [7]. One hope to increase the efficiency of the solid targets is to heat volumetrically the whole target through a laser bleaching wave [14].

We presents measurements of $\mathrm{x}$-ray output from laser-heated Ti K-shell emitters in a low-density-aerogel plasma. We have achieved a density of $\rho=3.1( \pm 0.1) \mathrm{mg} / \mathrm{cc}$ in our aerogel targets, which gives an ionized density relative to the laser critical density of $0.1 n_{\text {crit }}\left(n_{\text {crit }}=9 \times 10^{21} \mathrm{~cm}^{-3}\right)$. Previous work with aerogel materials saw only subsonic heating at a density of $0.6 n_{\text {crit }}$ [15]. Other works with low-density (organic) foams have ranged in density from $>0.1 n_{\text {crit }}$ to $\sim 0.7 n_{\text {crit }}$ [15-18], and have all seen transor subsonic heating and large fractions of the incident energy scattered by parametric instabilities $[16,17]$. The targets of the present work show supersonic heating, $\mathrm{x}$-ray output $1-2 \%$ of the incident laser energy, and minimal scattering losses. Details of the measurements of supersonic heating in these targets are presented in Ref. [19].

\section{EXPERIMENT DESCRIPTION}

These experiments were carried out on the OMEGA laser at the Laboratory for Laser Energetics (LLE), University of Rochester. Four targets, at two different densities, were shot at two different laser intensities. The targets for these experiments were doped $\mathrm{SiO}_{2}$ aerogels with an aggregate density of 3.1 or $3.3( \pm 0.1) \mathrm{mg} / \mathrm{cc}$; the $\mathrm{SiO}_{2}$ aerogel contained 3 atomic percent (atom\%) Ti. These aerogel targets, when fully ionized, had an electron density that is 8.9 or $9.8 \times 10^{20} \mathrm{~cm}^{-3}$ or $\approx 0.1$ times the critical density, $n_{\text {crit }}$, for $0.351 \mu \mathrm{m}(3 \omega)$ light. The final aerogel material was formed by super-critical extraction of the solvent used to mix the titanium and silicon alkoxides. The density of the aerogel produced is determined by gravimetric analysis of the bulk sample. Analysis by atomic absorption spectroscopy confirmed that the Ti remained at the 3 atom\% level after the solvent-extraction process. Visual inspection confirmed that the Ti was uniformly dispersed throughout the sample (no optical scattering centers or crystallites). These doped-aerogel targets are an important step in the development of low-density foams, which are of interest to ICF, shock physics and radiative transport experiments. The targets were cast in cylindrical Be tubes that had walls $80 \pm 5 \mu \mathrm{m}$ thick. The walls had a transmission $\geq 90 \%$ for the Ti K-shell x-rays $(h v>4.5 \mathrm{keV})$ of interest. The cylinders were $2.2 \mathrm{~mm}$ long with a $2.0 \mathrm{~mm}$ inner diameter, accurate to $3-5 \mu \mathrm{m}$. A schematic of our target, laser-beam cones and the diagnostics used to measure the $\mathrm{x}$-ray output from the target is shown in Fig. 1.

Twenty beams were used in three cones on each face of the the target; two shots had both cylindrical faces irradiated, two shots had irradiation on a single face (see Table 1). Beams cones were at $21.4,42.0^{\circ}$ and $58.9^{\circ}$ to the cylinder axis (henceforth, cone 1,2 and 3 , respectively). The cone 1 beams were focused to $200 \mu \mathrm{m}$ at a point 1100 $\mu \mathrm{m}$ from the face of the target in order to keep the beam footprints in the Be cylinder, the other two cones are focused to $300 \mu \mathrm{m}$ at the target face. Differential polarization rotators (DPRs) were employed to smooth all beams. The DPRs increased the stated 


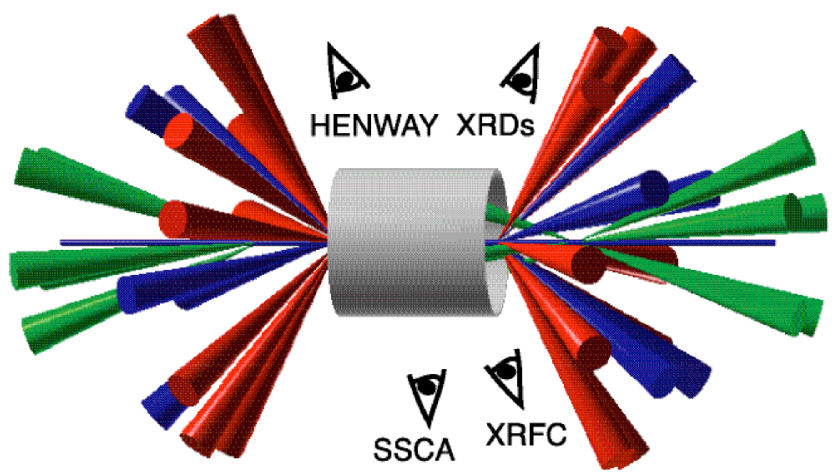

FIGURE 1. Schematic of our target, beam cones, and diagnostics used to measure the Ti x-ray output from the doped foam.

TABLE 1. A summary of the shots in this series of experiments. Columns are OMEGA shot number, target density, laser intensity at the target face, and faces irradiated.

\begin{tabular}{lccc}
\hline shot & $\rho(\mathrm{mg} / \mathrm{cc})$ & $\mathrm{I}_{\text {Las }}\left(10^{15} \mathrm{~W} / \mathrm{cm}^{2}\right)$ & Faces irradiated \\
\hline 31047 & 3.1 & 1.7 & 2 \\
31048 & 3.1 & 3.3 & 2 \\
31051 & 3.3 & 3.4 & 1 \\
31052 & 3.3 & 1.7 & 1 \\
\hline
\end{tabular}

size of the focal spot for each cone by $70 \mu \mathrm{m}$. Beams in each cone had a footprint at the target face of $\approx 440,475$ and $650 \mu \mathrm{m}$, respectively. The measured average energies in each beam for each two-sided and each one-sided shot were, 180.7, 362.6, 380.6 and $189.0 \mathrm{~J} /$ beam, respectively. One-ns-square pulses were used for irradiating the targets in these experiments. The laser intensities ranged from $I_{\text {Las }}=(1.7-3.4) \times 10^{15} \mathrm{~W} / \mathrm{cm}^{2}$ at the target faces. The dependence of our results on laser intensity and laser energy will be discussed below.

Several diagnostics were used to measure the Ti K-shell output from these targets. The primary measurement was made with a two-channel crystal spectrometer (HENWAY) onto direct exposure film (DEF), both channels employed pertaerthritol (PET) crystals. The spectrometer looked at the wall of the target at an angle of $119.1^{\circ}$ with respect to the cylinder axis. The known geometry of the spectrometer, crystal reflectivity (measured elsewhere [5]), the attenuation of the differential filters in front of the dispersive crystals, and the known response of the DEF [20] are used to compute the energy in a given spectral band. The films were digitized using a microdensitometer with a $22 \times 22 \mu \mathrm{m}^{2}$ step size. The K-shell spectrum for Ti from both channels is shown in Fig. 2. The signal in the $\mathrm{H}$-like $\mathrm{Ly}_{\alpha}$ line at $4.97 \mathrm{keV}$, as recorded on the higher-energy channel, and the 


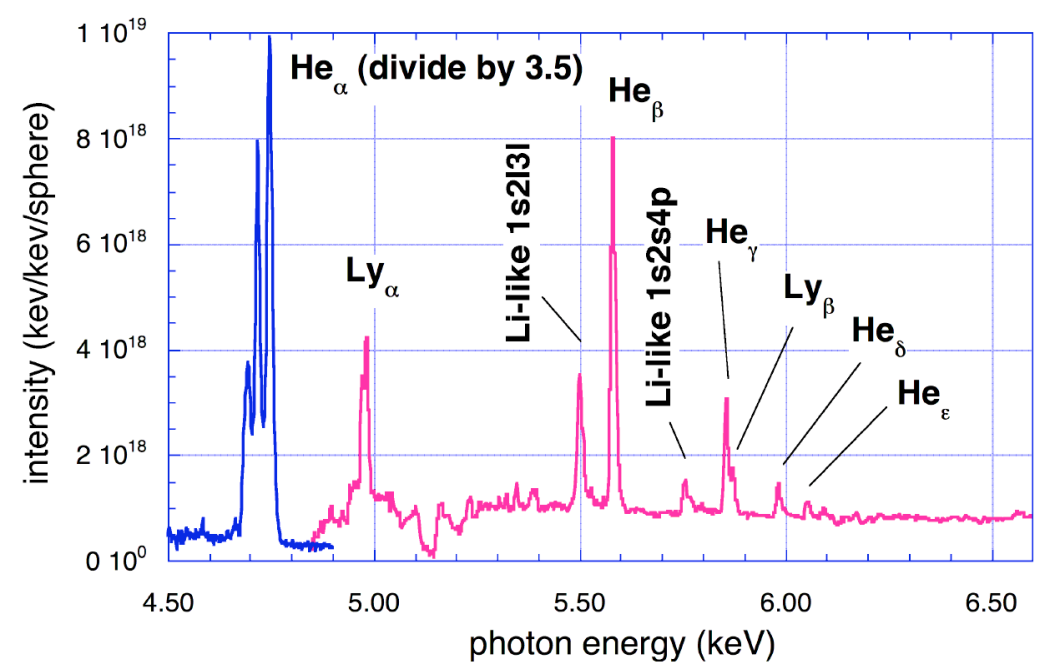

FIGURE 2. Ti K-shell spectrum, in absolute units, from the highest power shot (\# 31048) in our series. The output integrated from 4.67 to $5.0 \mathrm{keV}$ gives an output equal to $1.9 \%$ of the incident laser energy $(14.5 \mathrm{~kJ})$.

signal in the He-like $\mathrm{He}_{\alpha}\left(1 s 2 p \rightarrow 1 s^{2}, h v=4.75 \mathrm{keV}\right)$ band $(\Delta E=0.11 \mathrm{keV}$ centered at $4.73 \mathrm{keV}$ ) found as described below are used to compute the performance of our targets. These spectral windows are similar to what has been reported in the literature for other Ti K-shell x-ray production experiments [8-11].

Photometrically calibrated, filtered aluminum X-ray diodes (XRDs) were also fielded; they returned data on one shot. The diode array viewed the target at the same angle with respect to the cylinder axis as the crystal spectrometer. The diode array has six independent channels filtered to cover the range from 1 to $10 \mathrm{keV}$. The deconvolved signal from the appropriate channels gives the total power radiated in a given spectral band (see Table 2 below).

Two-dimensional images of the $\mathrm{x}$-ray emission front in our target were obtained by a gated $\mathrm{x}$-ray framing camera (XRFC). The XRFC was filtered with 16 mils of $\mathrm{Be}$, which gave images of (essentially) pure Ti K-shell emission. The camera images were taken through $50 \mu \mathrm{m}$ pinholes onto four strips of a MCP, each strip had three images taken approximately $200 \mathrm{ps}$ apart and integrated for $80 \mathrm{ps}$. The camera had a view at a $63.4^{\circ}$ angle with respect to the target axis. Two frames from a shot with two-sided illumination $\left(P_{\text {Las }}=14.5 \mathrm{TW}, I_{\text {Las }}=3.3 \times 10^{15} \mathrm{~W} / \mathrm{cm}^{2}\right)$ are shown in Fig. 3 . The right edge of the pinholes in Fig. 3 seems to be slightly blocked. The XRFC data are consistent with the rates of axial propagation for the $\mathrm{X}$-ray emission fronts found from filtered $\mathrm{x}$-ray streak camera (SSCA) data [19], and showed only minimal curvature of the $\mathrm{x}$-ray front as it moved down the tube.

Since we are interested in maximizing the output of our foam $x$-ray sources, we've measured the energy reflected from the targets with LLE's full-aperture backscatter (FABS) system. Energy scattered from the target back into the optics of one cone 2 and one cone 3 beam was measured with both a calorimeter and spectrally with a streak camera. The Stimulated Brillouin Scattering (SBS) signal on the steeper cone 3 beam is a factor of $2-5$ larger than on the cone 2 beam, which may be due to an interaction 

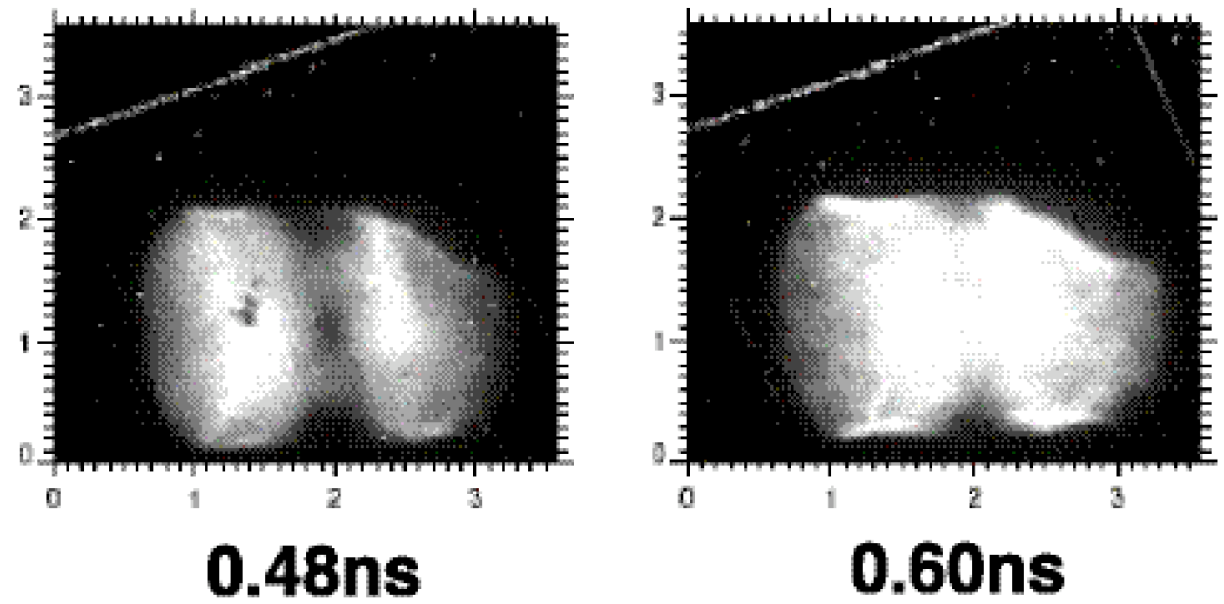

FIGURE 3. Frames at $\approx 480$ and 600 ps from the two-sided, high-power shot XRFC data that clearly show the two lobes of plasma before they meet at the center of the cylinder.

between the steeper cone 3 beam and the Be cylinder wall [21]. The two measurements for the Stimulated Raman Scattering (SRS) data are within 15-20\% of each other for three of the four shots. The calorimeter-measured energy in the SRS and SBS channels is averaged over the two beam cones. Note, measured energy is only the energy scattered into the F/6.7 optic of the two beams used, and our analysis assumes that the scattering on the shallow-angle cone 1 beams is well described by the average of the measured scattering on the cone 2 and 3 beams. The result is that the target reflects $2.4-5.2 \%$ of the incident energy. The aerogel of the present work is an open-cell foam with cell size estimated to be on the order of $40-50 \mathrm{~nm}$; to the $0.351 \mu \mathrm{m}$ laser pulse, the foam should appear approximately homogeneous $[15,16]$.

\section{SIMULATIONS}

We have used LASNEX [22] a 2-D, Lagranian, radiation-hydrodynamics code to simulate these targets. The simulations have three cones of beams onto either one or two faces of the foam cylinder at the appropriate angles. The simulations were run with the average experimental energy per beam and the measured pulse shape for each shot. The resulting peak intensities in the simulations ranged from $1.8 \times 10^{15}-4.0 \times 10^{15} \mathrm{~W} / \mathrm{cm}^{-2}$. Electron-heat transport in the targets is described by a multi-group flux-limited heat diffusion model based on the Spitzer-Härm thermal conductivity; a flux-limiter value of 0.1 gives the best agreement with the observed heat-front propagation [19]. A finite-element treatment of the electron-heat conduction has been employed, this gives a more accurate solution to the conduction equations for distorted Lagrangian meshes.

From the XRFC images (the high-power two-sided irradiation shot (\#31048) is shown in Fig. 3) we can see the X-ray emission fronts meeting at the target center by 600 ps after the start of the pulse. This is consistent with the meeting times predicted by LASNEX; 
TABLE 2. Results for X-ray CE measurements: column are shot numbers, measured UV energy delivered to the target, the $\mathrm{x}$-ray CE into $h v>4.7 \mathrm{keV}$ from DEF, CEinto $h v>4.0 \mathrm{keV}$ from the XRDs, and the prediction from LASNEX.

\begin{tabular}{lrrrr}
\hline shot & $\mathrm{E}_{\mathrm{UV}}(\mathrm{kJ})$ & $\begin{array}{r}\mathrm{CE}(\%) \\
\text { DEF }\end{array}$ & $\begin{array}{r}\text { CE }(\%) \\
\text { XRDs }\end{array}$ & $\begin{array}{r}\text { CE }(\%) \\
\text { LASNEX }\end{array}$ \\
\hline 31047 & 7.2 & 0.8 & - & 0.69 \\
31048 & 14.5 & 1.9 & - & 1.26 \\
31051 & 7.6 & 1.3 & - & 0.84 \\
31052 & 3.8 & 1.0 & 1.5 & 0.48 \\
\hline
\end{tabular}

the meeting times are later in the lower-power shots. From the gated XRFC images, we demonstrate a fast, volumetric heating of the targets.

The Detailed Configuration Accounting (DCA) atomic physics package was used by LASNEX to compute the radiative emission from silicon, oxygen and titanium. The DCA atomic physics package [23] solves rate equations for the population in each important excited state in each ionization state. This package is used when accurate atomic physics is needed for line diagnostics. A simple screened hydrogenic model produced the states and transition rates for the present simulations [21]. The optical depths computed for the $\mathrm{He}_{\alpha}$ line in these experiments are $\tau \lesssim 0.1$ for path lengths $\approx 1 \mathrm{~mm}$, so the system is optically thin to the $\mathrm{Ti} \mathrm{He}_{\alpha}$ X-rays.

\section{RESULTS}

Table 2 summarizes the x-ray output measurements for our four shots. In the table are listed the measured conversion efficiency relative to the listed energy of each shot (2nd column) found by integrating the $\mathrm{DEF}$ (3rd column) from the $\mathrm{H}$-like $\mathrm{Ti}^{21+} \mathrm{Ly}_{\alpha}$ transition at $\approx 4.98 \mathrm{keV}$ over a band $\pm 0.05 \mathrm{keV}$ wide, and adding the contribution for the $\mathrm{He}_{\alpha}$ $\left(1 s^{2}-1 s 2 p^{2} \mathrm{P}\right)$ complex plus its associated satellites. The contribution from the $\mathrm{He}_{\alpha}$ is found by integrating the signal in the $\mathrm{He}_{\beta}\left(1 \mathrm{~s}^{2}-1 \mathrm{~s} 3 \mathrm{p}{ }^{2} \mathrm{P}\right)$ feature, and calculating the ratio of $\mathrm{He}_{\alpha}$ to $\mathrm{He}_{\beta}$ with a detailed collisional-radiative model (CRM) [24, 25]. The ratio of energy in $\mathrm{He}_{\beta}$ to $\mathrm{He}_{\alpha}$ varies by $10 \%$ between 2 and $3 \mathrm{keV}$, the range of plasma temperatures predicted by LASNEX. The whole series of He-like and H-like lines visible in Fig. 2 are fit with a single-temperature spectrum; the ratio of $\mathrm{He}_{\alpha}$ to $\mathrm{He}_{\beta}$ from the model at that best-fit temperature is then used to compute the contribution of $\mathrm{He}_{\alpha}$ to the total Ti K-shell signal. Shots 31047 and 31051 were fit very well for $T_{e}$ $=2.25 \mathrm{keV}\left(\mathrm{He}_{\alpha} / \mathrm{He}_{\beta}=8.94\right)$, while shots 31048 and 31052 were fit at $T_{e}=3.0$ and $2.0 \mathrm{keV}\left(\mathrm{He}_{\alpha} / \mathrm{He}_{\beta}=8.53\right.$ and 9.47), respectively. The relative strength of $\mathrm{He}_{\alpha}$ to $\mathrm{He}_{\beta}$ is then checked for consistency with the Ti spectrum measured in the lower-energy channel of the spectrometer. The ratios of $\mathrm{He}_{\alpha}$ to $\mathrm{He}_{\beta}$ observed in the lower-energy channel are within $\approx 40 \%$ of these numbers. As stated above, the measured scattering energy losses 


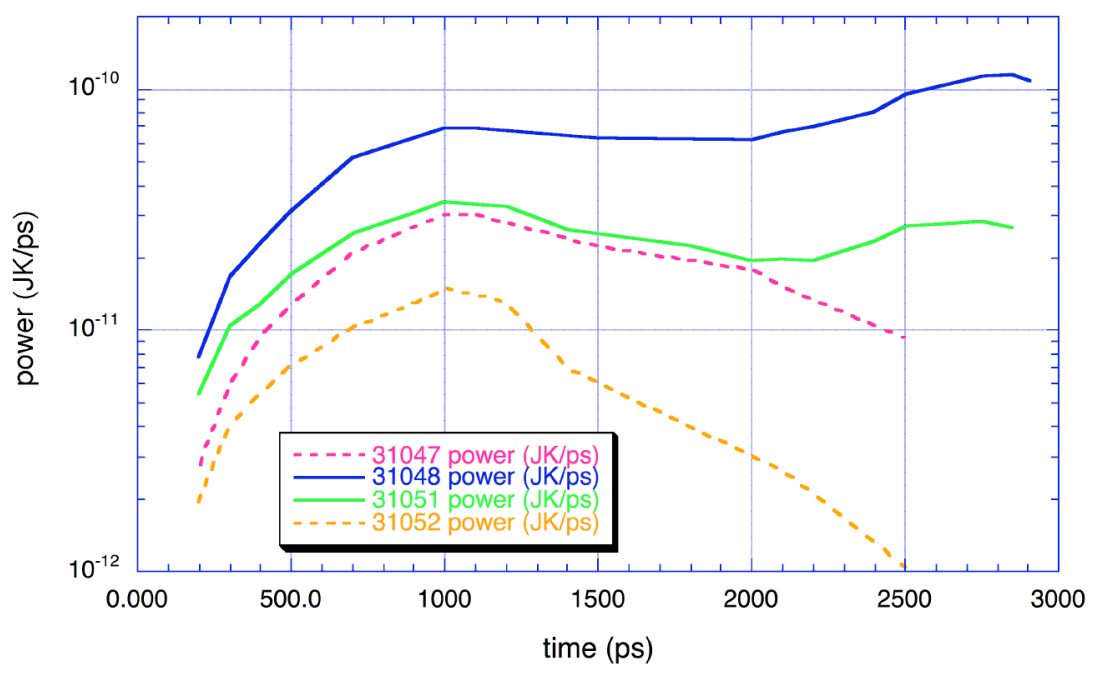

FIGURE 4. X-ray power (in JK/ps or $10^{9} \mathrm{TW}$ ) in the Ti K-shell band predicted for the four shots of the present work.

from the targets are $\lesssim 5 \%$. The CE numbers in Table 2 reflect this small correction to the incident laser energy.

There is an overall $\pm 30 \%$ uncertainty on the CE numbers measured with the DEF that results from measured uncertainties in crystal reflectivity across the width of the crystal [5], relative transmissivity of differential filters, and the level of film background. The signal measured with the XRDs in the band from 4 to $6 \mathrm{keV}$ for shot 31052 is also listed, there is a $\pm 20 \%$ error bar on the deconvolved XRD signal. The XRD signal contains the contribution from the higher- $n$ members of the He- and H-like Rydberg series, aside from $\mathrm{He}_{\beta}$, their contributions are negligible. The contribution from $\mathrm{He}_{\beta}$, based on our CRM, is $\approx 10 \%$. The measured CE for shots 31047 and 31051, given the $\pm 30 \%$ uncertainty quoted, were $\approx 1 / 2$ that of \# 31048 , this scales directly with the total energy delivered to the target. However, there is not a drop in CE for \# 31052, which had $\approx 50 \%$ of the energy of 31047 and 31051 . The laser intensity on each face of the target was a factor of two greater in \# 31051 than \# $31047\left(3.4 \times 10^{15}\right.$ versus $1.7 \times 10^{15}$ $\mathrm{W} / \mathrm{cm}^{2}$ ), the resulting CEs seem only slightly dependent on intensity in this case.

Also listed in Table 2 is the prediction for the x-rays out from our targets from LASNEX; the Ti spectrum in the simulation is integrated from $4.4 \mathrm{keV}$ up to $5.1 \mathrm{keV}$, thus including the $\mathrm{He}_{\alpha}$ and $\mathrm{Ly}_{\alpha}$ lines. The CE numbers from the DCA runs are listed in the last column of Table 2; output in the $4.67-5.0 \mathrm{keV}$ band is integrated in the simulation to $3 \mathrm{~ns}$. In every case, the targets appear to perform better than predicted by the simulations.

Figure 4 shows the power in the $4.5-5.1 \mathrm{keV}$ band predicted by our simulations over 3 ns. For the two lower-irradiance shots, the power falls off smoothly after 2 ns, while for the two higher-power shots (one and two sided), the power increases after $2-2.2 \mathrm{~ns}$. This is due, in the simulations, to the Be walls of the target crashing onto the target's axis and compressing the hot matter there. This type of ablatively-driven radial compression is different than, and comes later than, the axial compression in the two-sided irradiation 

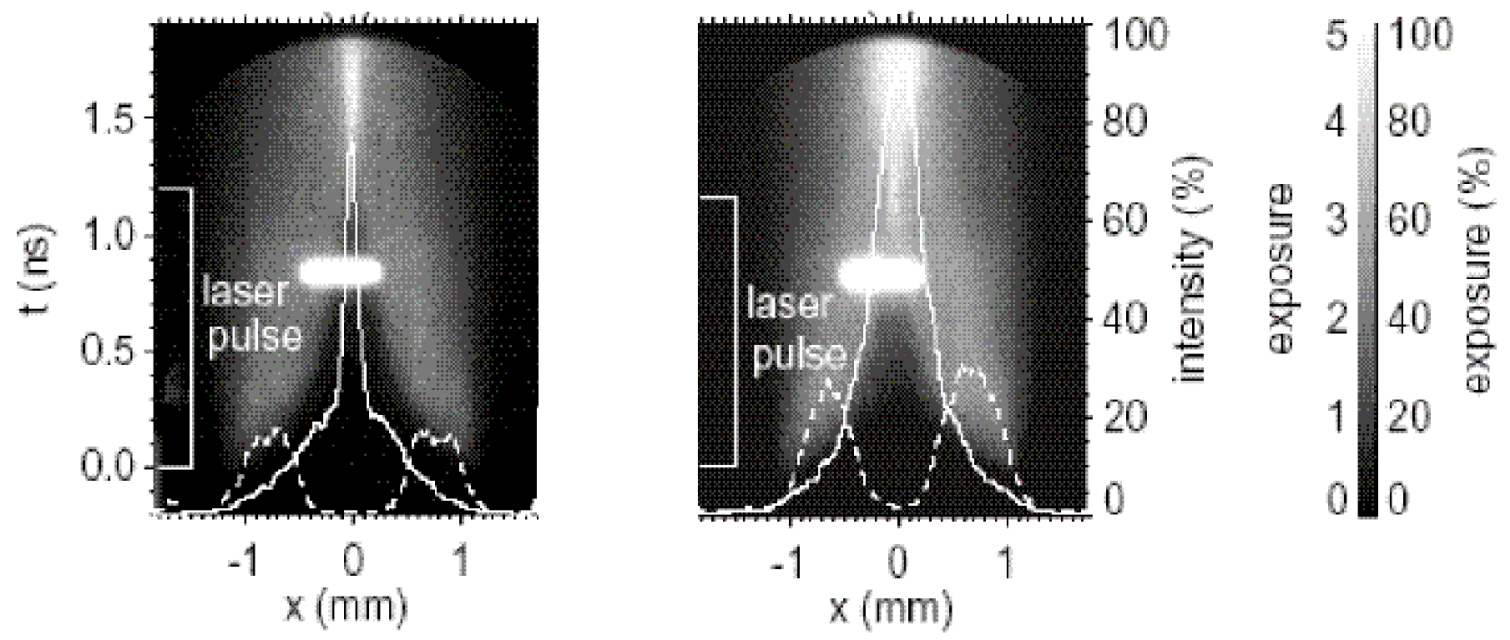

FIGURE 5. Streak measurements in targets irradiated from both sides at (left) lower energy, $7.2 \mathrm{~kJ}$, and (right) higher energy, $14.5 \mathrm{~kJ}$. Lineouts at $0.3 \mathrm{~ns}$ after the laser pulse starts (dotted) and $1.5 \mathrm{~ns}$ (solid) are plotted in each picture. The intensity scale for the lineouts is mapped on the right-hand side. The intense rectangular spot in the center is caused by $\mathrm{x}$-rays falling directly onto the photocathode.

cases discussed in the section below.

\section{HEAT WAVE PROPAGATION}

Figure 5 shows the images from an x-ray imaging streak camera (SSCA) for the two sided irradiation shots (31047 and 31048). The SSCA was fielded at a near side-on view $\left(79^{\circ}\right)$ and filtered for the Ti He-like and H-like lines in the 4.7-5.5 keV range using the K-edge of a vanadium filter [19]. The axial target dimension runs along the bottom of each image, and time goes upwards. The laser heat waves can be seen propagating from the LEHs towards the center of the target. The time origin was chosen as the time when emission is first observed. The heat front position is marked by the x-ray emission of the Ti dopant in the aerogel, corresponding to the axial location where the material was heated to temperatures high enough for Ti K-shell excitation. The time of collision between the converging fronts at the center of the target varies with the input laser energy, i.e. it is $1.2 \mathrm{~ns}$ for the low-energy case and $0.6 \mathrm{~ns}$ for the higher one. All images from the streak camera were recorded onto photographic film; for each image the relative intensity was obtained by converting the film optical density into areal energy density (exposure) using calibration wedges exposed onto each film. An earlier emission front with $2 \%$ intensity level, propagating ahead of the main heat wave is observed in all images. It is more pronounced and clearly faster in the higher-energy cases. The cause for this weak emission could be the intense heating at the LEH, where the strong beam focusing contributes to generation of energetic electrons that stream ahead and preheat deeper material. This indicates that a non-negligible fraction of the heat is transferred in front of the bulk electron flux and a careful treatment in calculating the energy transport is required at this point $[19,26]$. 


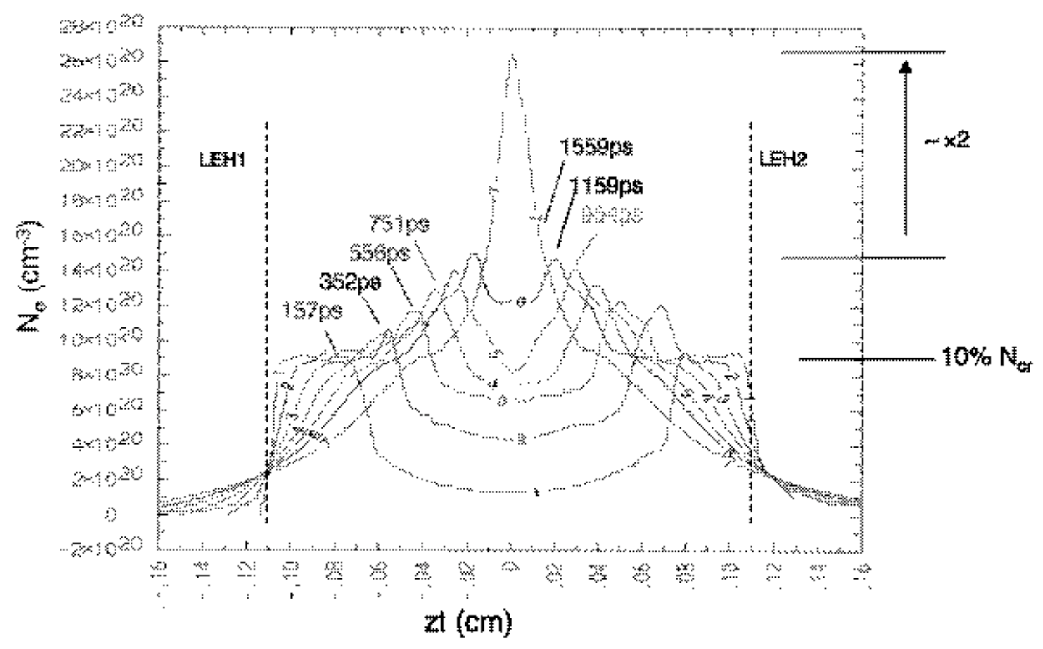

FIGURE 6. Density lineouts along the target axis from the LASNEX simulation for shot $31047(7.4 \mathrm{~kJ}$, $I_{\text {Las }}=1.7 \times 10^{14} \mathrm{~W} / \mathrm{cm}^{2}$ ) at different times.

The lineouts superimposed on the streak images in Fig. 5 show the profile of the emission intensity at two times before and after the wave interpenetration, i.e. $t=0.3$ and $1.5 \mathrm{~ns}$. We observe a change of the maximum emission intensity by more than would result in the case of a simple radiation overlap on the detector. Based on an examination of the simulated density and pressure distributions, we believe that the matter compression was responsible for this increase in emissivity. Density lineouts on the target axis from the simulation for shot 31047 are shown in Fig. 6. In the simulation, the electron density is three times the $0.1 n_{\text {crit }}$ level at $1.5 \mathrm{~ns}$, which is after the heat waves have interpenetrated. In the one-sided illumination cases, where the material has one direction for relaxation towards the unheated part of the target, the compression does not stagnate.

\section{SUMMARY AND DISCUSSION}

In summary, this paper reports on output of $\approx 1.0-2.0 \%$ of the incident laser energy (up to $\approx 15 \mathrm{~kJ}$ ) in the $4.67-5.0 \mathrm{keV}$ band, measured with a $\mathrm{x}$-ray crystal spectrometer, from a low-density Ti-doped aerogel target. Output of $1.5 \%$ is measured in the $4-6 \mathrm{keV}$ band with filtered x-ray diodes. The targets contained 3 atom $\%$ Ti in $\mathrm{SiO}_{2}$ aerogel, which when fully ionized had electron densities in the range $0.1 n_{\text {crit }}$. Supersonic, volumetric heating of the targets results in efficient conversion of the laser energy into x-rays. Supersonic heating of the targets is confirmed by analysis of heat-front propagation measured with a streaked x-ray imager [19]. The supersonic heating by the laser-drive beams is complimented in our low-density targets by low levels $(\lesssim 5 \%)$ of laserscattering energy losses.

Analysis of the propagation images shows a low-level $(\approx 2 \%)$ pre-heating front that races ahead of the bulk-driven $\mathrm{x}$-ray emission. This indicates a non-negligible amount 
of energy is transferred in front of the bulk electron flux, and a careful treatment of heat transport in the targets, using a non-local, non-linear model is required [19, 26]. Two-dimensional radiation-hydrodynamic simulations of the target explain strong brightening of the $\mathrm{x}$-ray emission when the propagating heat fronts interpenetrate, and suggest that the targets may undergo strong late time compression from laser-driven ablation of the target walls.

Finally, we note that previous work has found CEs for Ti targets that ranged from $0.01-0.6 \%$ [8-11] for lasers at $2 \omega$ and $3 \omega(0.53$ and $0.35 \mu \mathrm{m})$ with pulse lengths 100 -500 ps. The targets of the present work were irradiated with ns-scale pulses, these targets performed between $50 \%$ and a factor of three better than the results reported by Yaakobi et al. [8], which are the highest published CEs for Ti that we have found. The improvement in performance for our targets compared to those in in Yaakobi et al. scales nearly linearly with the increase of laser intensity above that in Yaakobi et al. Much work remains to be done developing and optimizing the low-density, dopedfoam targets as bright, efficient $\mathrm{X}$-ray sources. We have recently shot $\mathrm{Zn}$-doped foams at OMEGA, which emit strongly at $h v=9 \mathrm{keV}$, and analysis is underway.

This work was performed under the auspices of the U.S. Department of Energy by University of California Lawrence Livermore National Laboratory under contract No. W-7405-Eng-48.

\section{REFERENCES}

1. N. C. Woolsey, D. Riley, and E. Nardi, Rev. Sci. Instru. 69, 418 (1998).

2. F. Ze et al, J. Appl. Phys. 66, 1935 (1989).

3. S. G. Glendinning et al, Phys. Plasmas 7, 2033 (2000).

4. S. R. Goldman et al, Phys. Plasmas 6, 3327 (1999).

5. C. A. Back et al, Phys. Rev. Lett. 87, 275003, (2001).

6. R. Kauffman, Handbook of Plasma Physics, A. M. Rubenchik and S. Witkowski, eds., 3, 111, Elsevier, Amsterdam (1991).

7. R. Kodama et al, J. Appl. Phys. 59, 3050 (1986).

8. B. Yaakobi et al, Opt. Commun. 38, 196 (1981).

9. D. L. Matthews et al, J. Appl. Phys. 54, 4260 (1983).

10. D. W. Phillion and C. J. Hailey, Phys. Rev. A 34, 4886 (1986).

11. G. J. Tallents et al, J. Quant. Spectrosc. Radiat. Transf. 43, 53 (1990).

12. G. A. Kyrala et al, Appl. Phys. Lett. 60, 2195 (1992).

13. J. Workman and G. A. Kyrala, Rev. Sci. Instru. 72, 678 (2001).

14. J. Denavit and D. W. Phillion, Phys. Plasmas 1, 1971 (1994).

15. J. A. Koch et al, Phys. Plasmas 2, 3820 (1995).

16. K. A. Tanaka et al, Phys. Fluids 28, 2910 (1985).

17. H. Figueroa, C. Joshi, and C. E. Clayton, Phys. Fluids 30, 586 (1987).

18. V. V. Gavrilov et al, Quantum Electronics 31, 1071 (2001).

19. C. Constantin et al., Phys. Rev. Lett., in preparation, 2004.

20. B. L. Henke, et al, J. Opt. Soc. Am. B 3, 1540 (1986).

21. K.B. Fournier et al., in Laser-Generated and Other Laboratory X-Ray and EUV Sources, Optics, and Applications, ed. G. Kyrala, J.-C. Gauthier, C. MacDonald, A. Khounsary, Proc. of the SPIE 5196 (SPIE, Bellingham, WA, 2004) 194.

22. G. Zimmerman and W. Kruer, Comments Plasma Phys. Control. Fusion 2, 51 (1975).

23. Y. T. Lee, J. Quant. Spectrosc. Radiat. Transf. 38, 131 (1987).

24. S. H. Glenzer et al, Phys. Rev. E 62, 2728 (2000).

25. C. Biedermann, R. Radtke, and K. B. Fournier, Phys. Rev. E 66, 066404 (2002). 
26. G. Gregori et al, Phys. Rev. Lett., in press, 2004. 